\title{
Multi-agent platform for development of educational games for children with autism
}

\author{
Sjriek Alers \\ Eindhoven University of Technology \\ P.O.Box 513, 5600MB \\ Eindhoven, The Netherlands \\ s.h.m.alers@tue.nl
}

\author{
Emilia I. Barakova \\ Eindhoven University of Technology \\ P.O.Box 513, 5600MB \\ Eindhoven, The Netherlands \\ e.i.barakova@tue.nl
}

\begin{abstract}
Multi-agent system of autonomous interactive blocks that can display its active state through color and light intensity has been developed. Depending on the individual rules, these autonomous blocks could express emergent behaviors which are a basis for various educational games. The multi-agent system is used for developing games for behavioral training of autistic children. This paper features the functional and electronic design of the individual blocks and transformation of the multi-agent system to a platform that allows multiple games to be designed through easy reprogramming of the blocks. Three game concepts that show the type of games that can easily be implemented and reprogrammed are described. The impact of this platform is shortly mentioned in the discussion. The initial tests of using the platform for various educational games are very positive. However, the results of user tests go beyond the scope of this paper and are not discussed in the text that follows.
\end{abstract}

Keywords - multiagent interactive platform, tangible interaction, autism, game design.

\section{INTRODUCTION}

Multi-agent system research offers a possibility to incorporate autonomy and intelligence of the individual agents and therefore constitutes a promising paradigm for designing challenging games. The technologies used in game engines and multi agent platforms, however, are not readily compatible due to some inherent differences of the concepts. Where game engines propagate efficiency and centralized control, the multiagent platforms assume autonomy of the agents. This drawback is naturally resolved when the central control in the game is replaced by a human control, i.e. the multi-agent platform is used for games in which the control of the game is taken by humans. In this case the following problems need to be solved: are the actions synchronous, how agents communicate through the agent platform, how to monitor results of actions, what information is available for the agents from the game and how it is expressed in the agent rules.

Specifically we designed a multi-agent platform and games for children with autistic spectrum disorders (ASD). Children with ASD have an abnormal or impaired development in social interaction and communication [1]. Early behavioral or cognitive intervention can help children gain self-care, social, and communication skills. Poor social and communication skills can have far-going consequences for later participation in society. To develop these skills it is important that the autistic children are stimulated and challenged to communicate with others, we think that technology can contribute to this, because autistic children like technological gadgets and logical thinking. Robots, in particular, have been used for behavioral training for autistic children because of several reasons. First, autistic people like computerized games, predictable behavior, and repeating movements. In addition, developments in robotics during the past two decades have brought to autonomous or tele-operated robots that can act in unstructured environments. Pioneering work in using robots for autistic care has been conducted by Robins, Dauterhahn and colleagues, see for example [7],[8],[6]. The most recent developments in using robots for autistic care are based on human like robots that interacts with autistic children and a variety of interesting experiments has been reported in [8],[6],[9] among others. To avoid that the robot or other device with sensory -motor capabilities will even further isolate the child [7], an embodied toy that can be used by more than one child at the time is developed.

Inspired by observations, and discussions with teachers at autistic schools and institutions we have chosen a toy consisting of multiple interactive building blocks [2],[3]. Blocks are a usual toy of the autistic children. Equipping the blocks with sensory and expressive features will allow the children to play their usual games as well as there would be affordances for new games. In a broad sense the blocks are robotic agents, since they sense the environment and react by changing their color and intensity. Previously, LEGO building blocks have been used to enhance the social skills of autistic children [4],[5]. Similarly, we use building blocks. Differently, our blocks can interact by detecting their neighbors through the build-in sensors and show behavior in response to their sensory stimulation and learning algorithm. The internal organization of the blocks is of similar complexity as the existing commercial mini-robot [6], so in this respect this work relates to use of robots for training of autistic people.

Self-organization properties of the multi-agent block system can result in emergence of complex behaviors although the individual rules of all their agents are simple. By trying to compose the rules in such a way that the emergent behaviour of the overall system could be perceived as social, we hope to make this multi-agent system of interactive blocks comprehendible to the children and ultimately, a simulation of a simple society. The added feature of the platform is in the possibilities that a simple change of rules for the individual agent brings it to a different game. This makes the platform applicable for variety of purposes, ranging from a simple game 
to systematic behavioral training through games with increasing complexity.

\section{SYSTEM OVERVIEW}

A multi-agent system of interactive blocks that can communicate with each other and can display their individual active state to the outside world was developed, see Figure 1. Depending on the individual rules these interactive blocks could express many collective behaviors which can be a basis for various educational games.

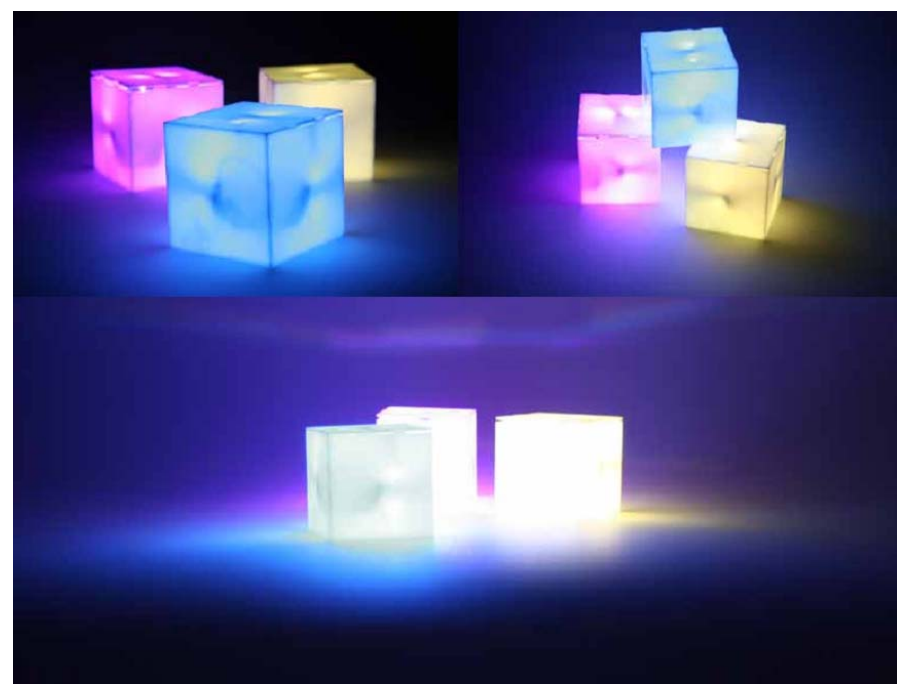

Figure 1. Interactive blocks.

Previous study [2],[3] has shown that constructing of patterns that configure themselves in an emergent but regular manner has a positive impact on stimulating explorative behavior, awareness of other players and turn-taking. The fascination of autistic children for patterns and regularity was shown to captivate their interest, and the emergently changing behavior might stimulate their motivation to try out new things. We have chosen cubes as a basic form, because regular structures can be built with them. Moreover, everyday practice in autistic schools shows that children like playing with cubes. The size was chosen so that the blocks with a cubic shape can easily be grasped by a child, but still is big enough to prevent a single child to "occupy" all the cubes. This choice was made to encourage children to join their efforts in building patterns together or at least make the child allow others to add to his construction, like another child or a caregiver.

Different states of the interactive blocks are expressed in light-color and -intensity. If an interactive block is in the vicinity of another block, the neighboring block detects the state and can adapt its own state according to the rule set implemented. To do this the blocks must be able to communicate with each other, but only if they are within the correct range and angle. Figure 2. shows how the blocks need to be positioned to be able to communicate. This interaction principle is the basis for the hardware and software design.

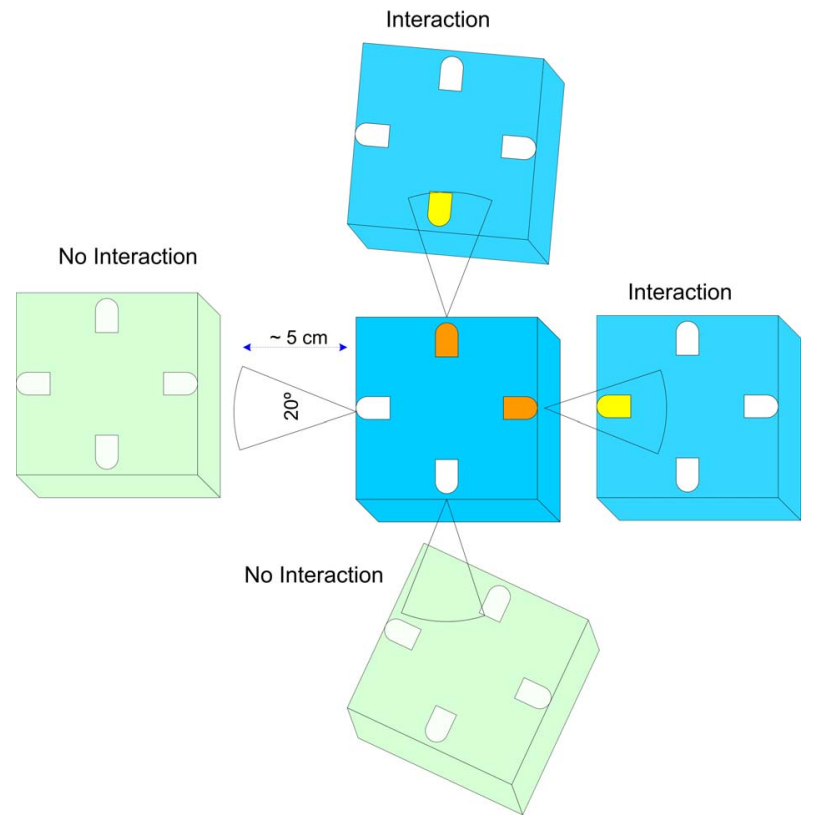

Figure 2. Block positioning

\section{A. Architecture}

Considering the design specifications the following functional stages can be determined: expression of the current state, communication between blocks, processing/deciding the new behavior, based on the sensed and communicated information and expressing the new behavioral state. All functionalities are interconnected as displayed in the system diagram in Figure 3.

Because static blocks behavior can be expressed effectively by color and intensity change we use this for expressing the current state of the interactive blocks. Moreover, autistic children like stimulation with dimmed light, and many regular patterns can be expressed through light. Using a series of wide angle Red Green Blue (RGB) Light Emitting Diodes (LED's), spread over the design makes it possible to create a dimmable homogenous light-field throughout the interactive blocks as is shown in Figure 1.

Communication between the blocks should only be possible at close range. Transmission by infrared light is a way to send data over a close range to an in-sight receiver; this implies that the transmitting and receiving interactive block have to be aligned what would be the case if they are placed in a regular pattern as can be seen in Figure 2. Every side of the interactive blocks must be able to transmit and receive data because the mutual orientation of the objects is unknown; therefore the infrared transmitters and receivers are mounted at every side of the blocks. The transmitters simultaneously send-out the agent's current state from all sides of the block. As sender, an infrared LED with a relative small exit angle is used to increases the requirement for the alignment of the blocks. Infrared is in another spectral range then the visible LED light, but to prevent any disturbances from inside or outside light, the outgoing data signal is modulated on a carrier frequency, to increase the reliability of the data transfer. Receiving of the neighbors infrared data-signal is done by six individual 
channels, one for every side of the block as displayed in the system diagram in Figure 3. The infrared receiving-modules pick up the modulated data signal, demodulate the signal and eliminate unwanted infrared disturbance. The received data will be passed through to the processing unit

The heart of the interactive block is a microcontroller that controls the timing of the data communication, processes the sensed data, makes decisions based on the game rules, and controls the expressed behavior.

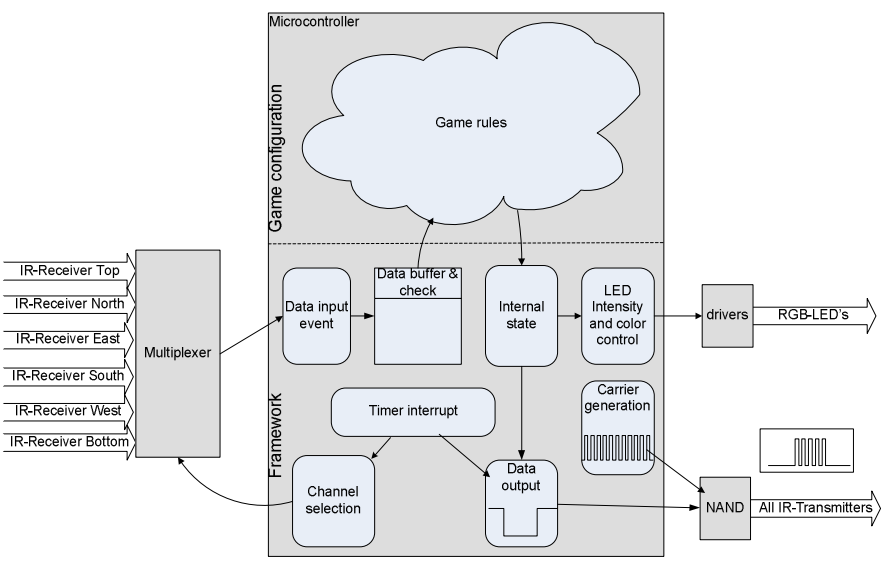

Figure 3. System diagram

\section{B. Software}

Within the microcontroller we setup a framework that controls the data communication, timing relationships and the Input/Output to the hardware. Within this framework the rule set of the actual game can be implemented. In Figure 3. the main functionalities are shown inside the microcontroller block.

Interfacing between the neighboring blocks is done using a serial data protocol, through the embedded Universal Asynchronous Receiver/Transmitter of the microcontroller, although this is normally used for peer to peer communication it is now implemented to send and receive data to the six sides of the interactive block. Sending is done simultaneously to all six sides, this is feasible because the data is the same for every side and represents the actual state of this interactive block. To match the demodulation frequency of the receiver modules, the data is modulated with the same frequency that is made by a timer controlled Pulse Width Modulation output. Receiving data of six receivers isn't possible at the same time so listening to neighbors-data is done each side after the other creating six different timeslots. There is no synchronization between the neighboring blocks, to ensure reliable communication the data is send to the receiver two times in every timeslot. Figure 4. shows the time slicing and the transmit- and listen bursts per timeslot. In order to avoid data-collisions as much as possible the data-burst is as short as possible.

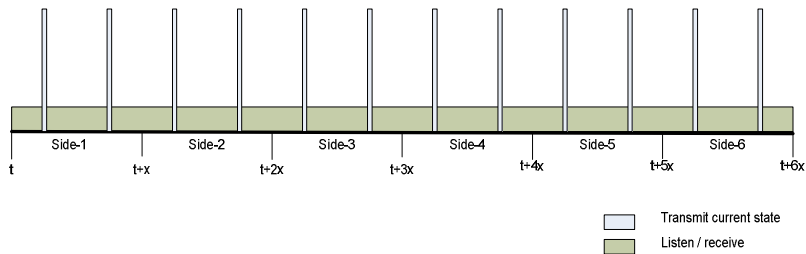

Figure 4. Data communication diagram

The absence of synchronization between the communicating blocks can cause data-collisions and so datacorruption, stray-light, reflections and objects that are on the edge of reception can also cause corrupted data. To avoid these drawbacks, some error detection measurements must be taken. We only use valid received data-packets that comply with the USART standard are received, all other packets are ignored. In the software an additional data-check is done to verify the validity of the received data and only valid data is taken into account. The receiving agent will only adapt to another state if data is received multiple times, this not only increases the reliability of the data-transfer but also ensures that a block that is not in the correct position but is moved beyond the agents reach will not be detected during game play.

Programming the games is done in the Microchip MPLAB programming environment and the programming language is C. It is rather hardware based programming because the program runs on a microcontroller and although the code to program the game rules can be seen as a separate part, it is still interwoven with the code for the physical interaction of the pins of the microcontroller.

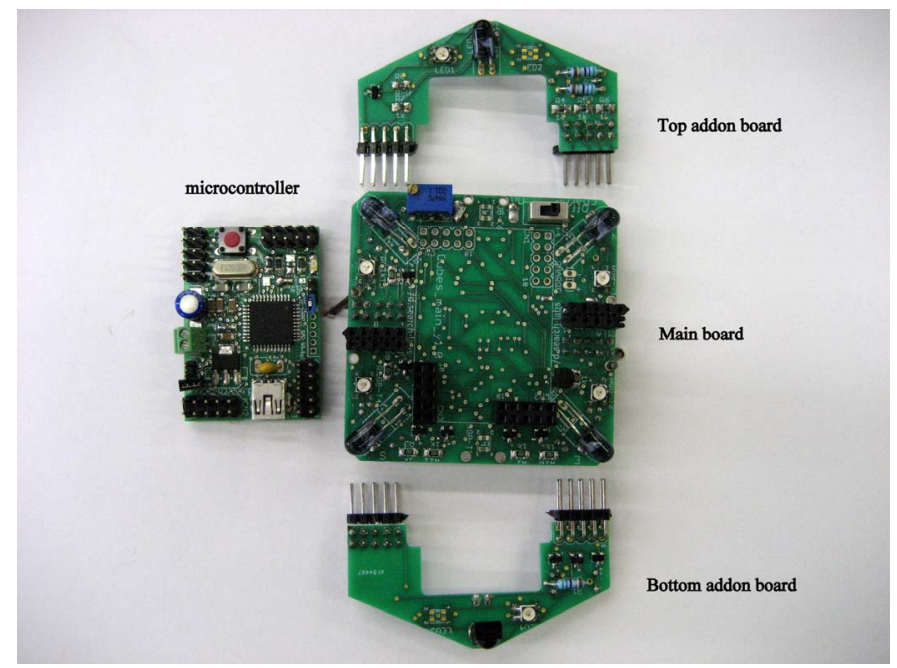

Figure 5. Electronic components

\section{Electronics}

The electronics of these agents are integrated on four custom made Printed Circuit Boards (PCB's). The boards; a microcontroller board, a main board and two add-on boards are shown in Figure 5.

A microcontroller platform based on the PIC18F4550 from Microchip is used. This 8-bit microcontroller platform that runs on an internal clock of $48 \mathrm{MHz}$ is powerful enough to execute 
the designated tasks. The ability to use Universal Serial Bus (USB) functionality in combination with a boot loader to reprogram the microcontroller over USB makes it a good prototyping platform for product-development.

The main board is a placeholder for the other boards and holds the electronics for four of the six communication channels. The microcontroller board and the add-on boards can be plugged onto this board. The electronics on this board are four infrared LED's, a modulator/inverter, four receiving modules, RGB-LED's, a battery charging circuit and a battery pack. In Figure 6. the electronic diagram can be seen.

The two add-on boards are similar in design and are place on top and on the bottom of the main PCB making this a real three dimensional PCB design, the completed design can be seen in Figure 7. On each of these boards one of the infrared sending LED is placed, one of the receiving units and there is room to place four of the RGB-LED's. See Figure 6. for the electronic diagram.

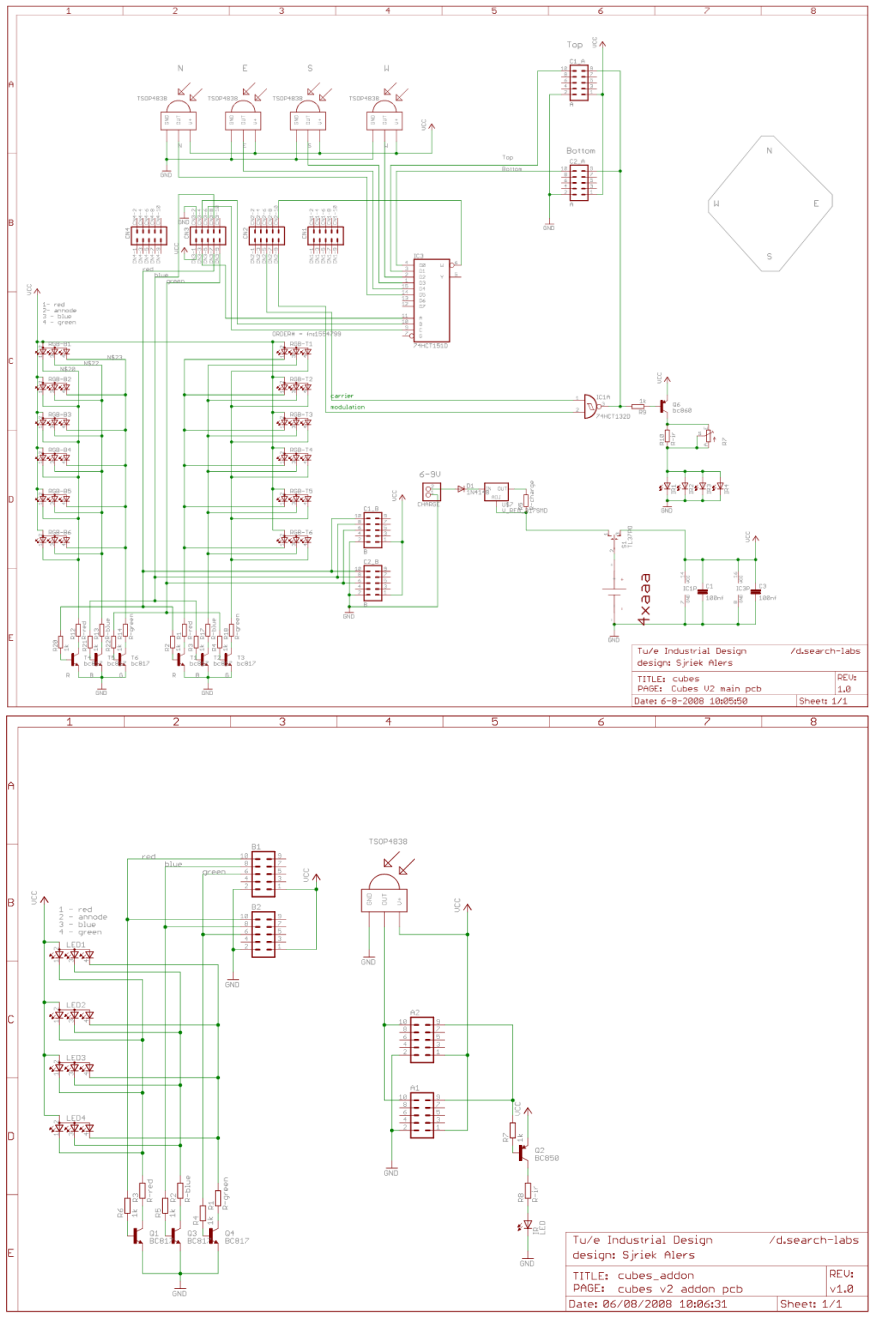

Figure 6. Electronic circuit diagrams

\section{Mechanics}

The mechanical casing of the interactive cubes is a fairly simple design and is made from six pieces of $2 \mathrm{~mm}$ thick white acrylic glass that, when put together, form a solid cube of $85 \times 85 \times 85 \mathrm{~mm}$. The white acrylic glass is chosen so that the technology inside is not visible to the user but the effects of the actions are visible, so the light color that is generated inside the cube is visible at the outside. The material also doesn't have any direct negative effects on the infrared transmission and reception except for some possible reflections. The cubes in Figure 7. show how the casing is build up and how the electronics fit into it.

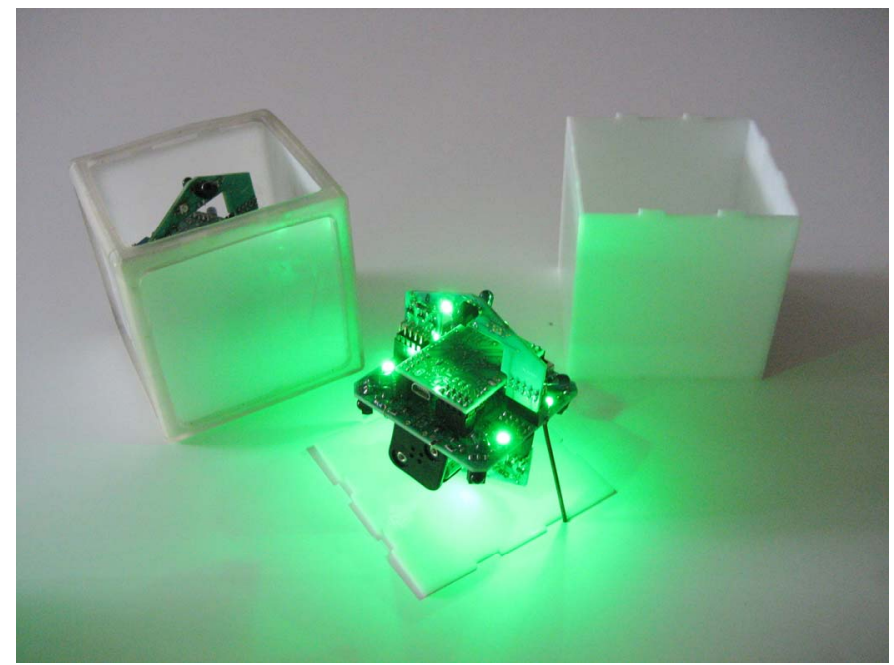

Figure 7. Casing and electronics

The casing is laser-cut and has reasonable sharp edges and pointy corners; to make it a safe toy to play with a siliconrubber protector is designed. This protector covers the hard and pointy edges of the acrylic glass and is placed on the left cube of Figure 7.

\section{GAME CONCEPTS AND IMPLEMENTATION}

We make a distinction between the platform and the specific games. By platform we mean the hardware, including form, sensors, actuators, microcontroller, and the programming environment, which allows different behaviors to be simulated. By game we mean a specific embedded program to make the blocks behave according to specified rules, together with the explanation of the rules as to be delivered to the players. The game/platform distinction makes it easier to develop several games and compare different games on the same platform.

We made a first step in designing a professional platform that can be used in training of autistic children and is derived from early prototype that have been tested in [2],[3],[10]. These prototyping platforms had, and so this platform has the ability to communicate via infrared, and is able to express its state by one changing light color. Differently, in the current paper we are not considering a particular problem that we want to solve through games. Instead, the focus of this paper is to make a multi-agent platform on which different games can easily be implemented, in accordance with the needs of the separate practitioners. We show 3 different game concepts that serve two major purposes: first, to illustrate the potential of the design platform to be easily reprogrammed for the new game and second, to be well fitted for promoting social behavior. 
The first concept was named "Stratego blocks". It is a variant of the "Mouse, snake, elephant" game, by which each animal can win over the other-mouse is stronger than the elephant, the snake is stronger than the mouse, and the elephant is stronger than the snake. The game is played through the embodied lighting blocks that take the color of the animal that they represent, which stimulates the interest of the children. It has strategic elements and assumes usage of a playfield. The logical/strategic elements provided an additional challenge for the children with autism. The goal of the game can be accomplished only if the children work together with peers. By diverting the child's attention from patterns and promoting success by achieving the end goals of the game the child is challenged to break through their patterns of repetitive behavior. The goal of the game is to reach the flag field of the opponent. Two children receive 8 blocks with a random ranking: mouse (purple), snake (green) and elephant (blue), meaning: the mouse defeats the elephant, elephant defeats the snake etc. The colors are only visible on the players' side of the blocks and have to be divided on their side of the board. When two blocks of opposing sides meet, the ranks are displayed through color and the strongest wins, or when it is a tie both loose. The blocks won't remain passive though, when one block isn't used for a longer time it starts to signal the child that it wants to be placed somewhere else. This forces the child to divert his or her attention to different parts of the playing field. Overall the peer to peer aim of the game provokes social interaction and common goals when working together. The following Figure 8. illustrates some elements of the game.

The microcontroller picks the random ranking value. When two blocks meet the infrared communication interchanges' ranking between the blocks and the microcontroller determines if the block lost or won. To enable that the colors are only visible on the players' side of the blocks, an extra control of one group of LED is required by reconnecting some of the LED's to additional microcontroller pins.

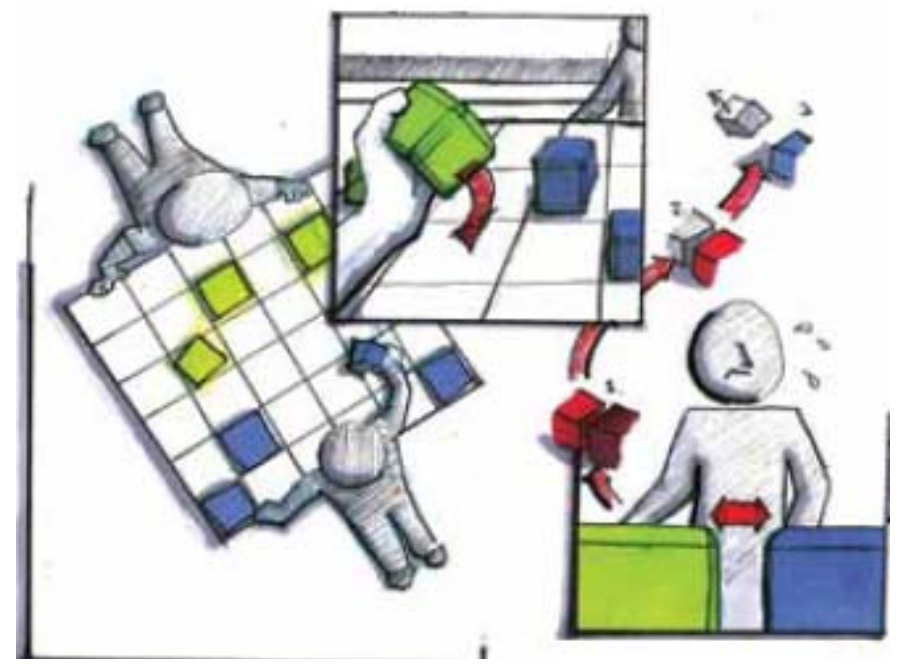

Figure 8. "Stratego blocks" game concept.

The second concept "No Place Like Home" focuses on expanding the context of play for the child, reducing his/her tunnel vision and improving social interaction with the other children. Children with autism display impairments in joint attention, which is their ability to coordinate the focus of attention on another person and an object of mutual interest. The goal of this concept is to divert the attention of the child towards the room. The blocks start in a default state and placement. Each of the blocks shows a belonging to a group through its color. On an abstract level this can be interpreted as belonging to a social group. Blocks that are socially weaker have a greater tendency to adapt to another color while stronger blocks contain the same color. The children change the position of the blocks, and over time the blocks start to form clusters and may "demand" a different space in the room. By blinking they show that they're not happy about their space and the child has to maneuver them through the room until they stop blinking which means the blocks are at ease (resemblance with "Hot/Cold" game). Overall the blocks will gradually increase the area of play for the child thus their interaction space within the environment is enlarged. While the game can be played with one person it has a higher entertainment value when played in cooperation with parents or peers. The following Figure 9. depicts the game scenario.

The microcontroller changes the color and intensity of the light of the blocks depending on the group it belongs to and the position inside the room. The Infrared communication enables the block to be aware of the surrounding blocks social setting.

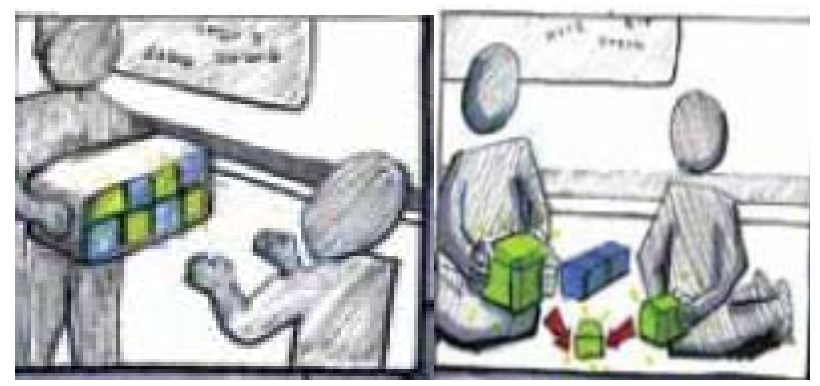

Figure 9. "No Place Like Home" game concept.

The third game concept was named "Kinetics". Children with autism show a higher prevalence of impairments within their gross and fine motor skills in comparison to typically developing children. Alleviation of this impairment is targeted with the "Kinetics" concept. The concept incorporates the essence of movement to the existing building blocks to improve the motor skills and challenge the children to explore different modalities in play. Each block represents an individual with certain strength. This strength is shown through light intensity. When two blocks are placed next to each other the block with weaker intensity will adapt its color to the stronger one. A second rule insures that larger amount of blocks will win over a smaller group. The child can shake the block in any direction and enhance its strength, empowering one block to overrule multiple blocks at once. The movements are also transferred to the light patterns and the way the other blocks change their colors, creating the illusion of spinning objects which has proven to be highly interesting for children with autism. Due to the multi-agent system properties interesting global behaviors and patterns can emerge. Games 
that can be played like "High Striker" or "Risk" are a natural way to add more purpose to these games. Overall the concept will provide an extra spatial dimension to the play and can train or record motor behaviors by the children. To what extend the children understand the affordance to change the colors of the blocks is not tested yet. The following Figure 10. illustrates two possible scenes from the game.

The microcontroller changes the intensity of the light of the blocks. To obtain the energy charging functionality, an accelerometer needs to be added to the existing hardware. The accelerometer also can give input for the changing light pattern. For creating the illusion of spinning objects, extra control of different groups of LED has to be made by reconnecting some LED's additional microcontroller pins.
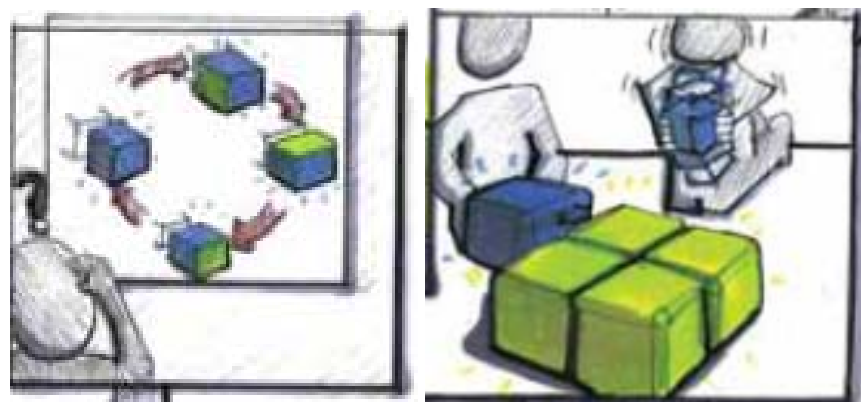

Figure 10. "Kinetics" game concept.

The implementation of the actual game is free to the gamedeveloper. This hardware platform and software framework is developed to be as multifunctional as possible. The second and third concepts resemble human social interaction. Changing between different games requires uploading a new set of rules to the blocks. In the second concept each block displays its need to be in a group through its color. Social weaker blocks have a greater tendency to adapt to another color while social stronger blocks don't. Weaker block can be recognized by a lower light intensity, but they become stronger when they are in a social group, their light intensity increases. When the blocks are grouped for too long they start demanding a new social setting and their lights start blinking. When they are placed into another social setting they stop blinking and will display their social strength again. The basic functionality of this game concept is described in the code below.

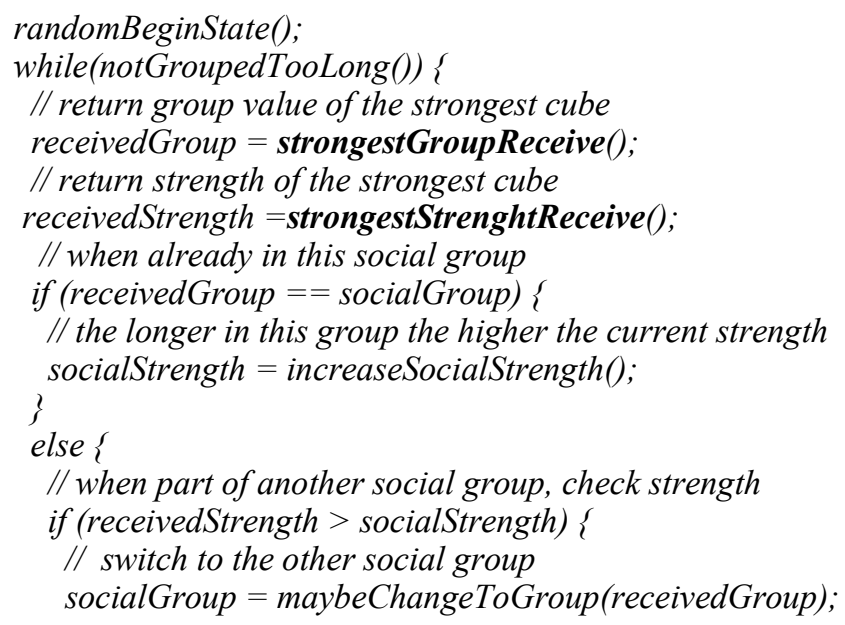

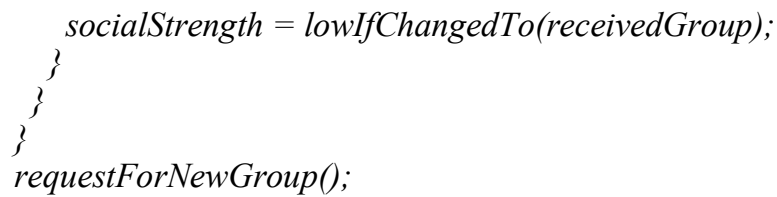

By reconfiguring some basic rules of the agents we can easily change this game into the third concept and let each block be an individual with a certain social strength that is shown through the light intensity. When two blocks are placed next to each other the weaker one will adapt its color to the stronger one. Now in addition a larger group of blocks will win over a smaller group. This game concept is described in the code below.

randomBeginState();

while(notGroupedTooLong()) \{

// returngroup value of the largest group

receivedGroup = largestGroupReceive();

// return strength of the largest group

receivedStrength = largestStrenghtReceive();

// when already in this social group

if (receivedGroup $==$ socialGroup) \{

// the longer in this group the higher the current strength

socialStrength = increaseSocialStrength();

\}

else \{

// when part of another social group, check strength

if (receivedStrength $>$ socialStrength) \{

// switch to the other social group

socialGroup = maybeChangeToGroup(receivedGroup);

socialStrength = lowIfChangedTo(receivedGroup);

\}

\}

\}

requestForNewGroup();

As can be seen from the previous two examples only the functions to determine the strength and state of the surrounding blocks have to be changed to go from the basics of one game concept to another. So only a small adaptation in the rules of the individual agents changes the outcome of the complete game.

\section{FUTURE DESIGN CONSIDERATIONS AND DISCUSSION}

During the game concept development additional preferred hardware features turned up. One of them was the ability to measure the orientation of the interactive cube and another was the possibility to "shake" the cube to change to another active state. These two features will be available if the platform also includes a three dimensional accelerometer. Another wishful feature would be that all LED's inside the interactive cubes could be controlled individually so that "light flows" could arise inside a cube. Other possibilities for future designs would be an even more strict separation between game implementation and hardware/communication functionality; this could be done by implementing a second microcontroller in the design. One controls all available hardware features and communication protocols and that is not re-programmable by the game developer. The game development will be done on a second microcontroller; the game developer has complete 
freedom to programs this and can receive data and give commands to the other microcontroller. In a next version it would be nice to be able to change the game rules without taking the prototype apart, so wireless communication could be wishful. For the ease of game programming and wireless communication an Arduino microcontroller platform in combination with a wireless chip as in [15] could be very suitable. The final product should have no visible interface or buttons anymore. For this wireless charging of the battery pack is needed and the possibility to switch the block on/off without a physical switch.

Earlier prototypes of the platform with somewhat simpler functionality was used to develop social training scenarios and games for behavioral training of autistic children [2],[3],[10]. The games have been co developed with medical practitioners from autistic caregiver institutions and after that tested with the children. Being exposed to the blocks, children showed increase in their explorative and some aspects of their social skills. Autistic children enjoyed very much the games because of their affinity to discover patterns, regularity and rules. Using this feature of the emergent toy we designed the games in order to provoke discussions and need to cooperate, i.e. different social behaviors. The games were tested with autistic children and the results were documented and published in [2],[3],[10]. This caused the necessity to redesign the blocks into the current platform that requires minor reprogramming and no basic hardware change when a novel game is developed. We aim at making these blocks a universal tool for training social skills to autistic children.

Our platform have been one of the inspirational sources of a research lines called Open ended play, and Decentralized interactive environments, which further developed independently and in parallel and has shown a very interesting game applications. This research is inspired by theories of situated action and theories about situated action [11],[14] and about emergent behavior in decentralized systems [13]. Instead of designing for goal-directed behavior, as is assumed by, for example, Norman's action cycle [12], the situated actions model assumes that players do not structure their activity beforehand, but that activity grows as the interaction in the context of use occurs. Bekker at al. [14] assume that by providing local interaction opportunities as a support for situated actions overall play patterns will develop.

\section{ACKNOWLEDGMENTS}

We would like to thank Gerrit-Willem Vos for developing several game concepts and testing them. Chet Bangaru we would like to thank for his mechanical expertise and the design of the silicone-rubber protector. Also we would like to thank Gilles van Wanrooij, Martnick Menting, R. van Limpt, Jelke Schippers, and Stijn Copieters for taking part in the initial block design. We are grateful to the /d.search-labs of the faculty of Industrial Design for providing the facility's and time to design and build these prototypes.

\section{REFERENCES}

[1] American Psychiatric Association, Diagnostic and Statistical Manual of Mental Disorders. American Psychiatric Association, Washington D.C., 1994.

[2] E.I. Barakova, G. v. Wanrooij, R. v. Limpt, and M. Menting, "Using an emergent system concept in designing interactive games for autistic children," in Proceedings of IDC7: ACM digital library.

[3] E. I. Barakova, J. Gillesen, and L. Feijs, "Use of goals and dramatic elements in multi-agent platform for behavioral training of children with ASD," in Proceedings of $I D C 08$, to appear in the ACM digital library.

[4] LeGoff, D.B., Use of LEGO@ as a Therapeutic Medium for Improving Social Competence, (2004) Journal of Autism and Developmental Disorders 34: 557-71.

[5] Owens, G. Humphrey, A. and Baron-Cohen, S. LEGO ${ }^{\circledR}$ therapy and the Social Use of Language Programme (SULP): ARC, CUERR Vol. 1 (3), pp. 143-149, July 2006. ISSN 1990-3839 @ 2006

[6] Billard, B. Robins, K. Dautenhahn, J. Nadel (2006) Building Robota, a Mini-Humanoid Robot for the Rehabilitation of Children with Autism . RESNA Assistive Technology Journal.

[7] Dautenhahn, K., Werry, I., Towards interactive robots in autism therapy (2004) Pragmatics and Cognition 12:1, 1-35.

[8] Robins, B, P. Dickerson, and K. Dautenhahn, "Robots as Embodied Beings - Interactionally Sensitive Body MovementsIn Interactions Among Autistic Children and a Robot," Proc. RO-MAN 2005

[9] Nadel, J. "Early Imitation and a Sense of Agency," Proc. 4th Intl. Workshop on Epigenetic Robots, 2004

[10] Barakova, E.I., Gillessen, J. Feijs, L., Social training of autistic children with interactive intelligent agents Jounal of Integrative Neuroscience, Vol. 8 No. 1, March 2009.

[11] Lave, J. (1988) Cognition in practice. Cambridge: Cambridge University

[12] Norman, D. (1990) The design of everyday things, New York: Basic Books.

[13] Resnick, M. (1997) Turtles, Termites and Traffic Jams Explorations in Massively Parallel Microworlds, MIT Press, Cambridge Massachusetts. Press.

[14] Bekke, T, Sturm, J, Eggen, B., Designing Playful Interactions for Social and Physical Play, Personal and Ubiquitous Computing, inpress, 2009.

[15] Alers, S., Hu, J. (2009). AdMoVeo: A Robotic Platform for Teaching Creative Programming to Designers. In M. Chang et al. (Eds.), Edutainment 2009, LNCS 5670 (pp. 410-421). Springer-Verlag Berlin Heidelberg 2009. 